VEIGA, RK; GONTIJO, LA; MASIERO, FC; MAAS, L; ODORIZZI, W. 2017. Caracterização de acidentes com tratores de rabiças no cultivo da cebola em Santa Catarina. Horticultura Brasileira 35: 124-128. DOI - http://dx.doi.org/10.1590/S0102-053620170119

\title{
Caracterização de acidentes com tratores de rabiças no cultivo da cebola em Santa Catarina
}

\author{
Ricardo K Veiga ${ }^{1}$; Leila A Gontijoㅜ; Fabrício C Masiero²; Larissa Maas²; Willian Odorizzi² \\ ${ }^{1}$ Universidade Federal de Santa Catarina (UFSC), Florianópolis-SC, Brasil; ricardokveiga@bol.com.br; leila@deps.ufsc.br; ${ }^{2}$ Instituto \\ Federal Catarinense (IFC), Rio do Sul-SC, Brasil; masiero@ifc-riodosul.edu.br; larissamaas@ifc-riodosul.edu.br; odorizzi_w@hotmail. \\ com
}

\section{RESUMO}

A horticultura catarinense representa importante papel na economia do estado. Como hortaliça de maior destaque econômico, a cebola tem sua produção concentrada nas pequenas propriedades rurais. $\mathrm{O}$ aumento de produtividade só foi possível com a utilização de maquinário agrícola versátil e de baixo custo. Os tratores de rabiças foram instrumentos que permitiram esse avanço e de forma indireta mantiveram o horticultor no campo. Mas a utilização intensiva dessas máquinas sem cuidados essenciais na sua operação trouxe reflexos negativos para a saúde destes trabalhadores. Evidenciou-se o aumento na quantidade de acidentes nas últimas décadas. Este número é pouco preciso e as causas desconhecidas, uma vez que não são computados nem mesmo como acidentes de trabalho. Esta pesquisa busca caracterizar causas dos acidentes com tratores de rabiças no contexto do cultivo da cebola, identificando o perfil do acidentado e em que circunstância ocorreu o acidente. Para isso foi utilizada pesquisa tipo levantamento (survey) através de questionários semiestruturados respondidos por 79 agricultores. A ferramenta estatística foi a correlação entre variáveis, por meio do coeficiente de Pearson, a uma significância de $0,1 \%$. Foram investigadas as correlações entre as variáveis: faixa etária do acidentado, potência do trator, membro afetado, severidade do acidente, causa genérica e causas específicas. Constatou-se que a faixa etária mais suscetível foi dos 10 aos 20 anos $(21,7 \%)$, a causa genérica foi atitude insegura $(60,8 \%)$ e as causas específicas mais citadas foram falta de atenção $(28,0 \%)$, perda de controle em aclive/declive (23,3\%), falha mecânica (12,7\%), falta de proteção $(11,3 \%)$ e operação da máquina em condições extremas $(10,7 \%)$. As correlações mais significativas foram entre a idade e a atitude insegura e a idade e falta de atenção.

\begin{abstract}
Accidents with power tillers used for onion cultivation in Santa Catarina State, Brazil
\end{abstract}

Santa Catarina family farming plays an important role in the state's economy. Products such as onion have its centralized production in small farms that employ family labor. The increase in productivity in these small plots was only possible with the use of versatile agricultural machinery. The power tillers were instruments that enabled this progress and indirectly kept the farmer in the field. But, the intensive use of these machines without essential care for their operation can bring negative effects to the farmer's health. The growth of machine fleet and the need to expand production may explain the increase in the number of accidents with these machines in recent decades. This number is doubtful and the causes unknown, because they are not computed as work accidents. This research aims at characterizing the accidents, identifying the machines, the victim's profile and the circumstances in which they occurred. This research used semistructured questionnaires answered by 79 farmers. The statistical analysis used was the descriptive and variance analysis using Pearson's coefficient in the correlations. Possible correlations between the following variables were investigated: age of the victim, machine power, affected limb, severity of the accident, general and specific cause. The most susceptible age range was from 10 to 20 years $(21.7 \%)$ the general cause was insecure act $(60.8 \%)$ and the most cited specific causes were lack of attention (28.0\%), loss of control uphill/downhill (23.3\%), mechanical failure (12.7\%), lack of protection $(11.3 \%)$ and machine operation in extreme conditions $(10.7 \%)$. The most significant correlations were between the age and unsafe act and the age and lack of attention.

Keywords: Allium cepa, safety.

Palavras-chave: Allium cepa, segurança.

(Recebido para publicação em 29 de fevereiro de 2016; aceito em 8 de julho de 2016) (Received on February 29, 2016; accepted on July 8, 2016)

\begin{abstract}
A agricultura familiar tem grande importância econômica e social no espaço rural brasileiro. Segundo FAO/ INCRA (1994), a agricultura familiar tem como característica básica a íntima relação entre trabalho e gestão, onde a direção do processo produtivo é conduzida pelos proprietários. A agricultura familiar lançou mão da mecanização
\end{abstract}

agrícola para se tornar mais rentável. Uma das máquinas que possibilitou este avanço foi o trator de rabiças. Sua versatilidade e baixos custos de aquisição e operacional o popularizou entre os horticultores. Segundo ANFAVEA (2014), a produção de tratores de rabiças atingiu seu pico em 1986 quando foram comercializadas mais de sete mil unidades. Atualmente em Santa Catarina existem aproximadamente 20 mil tratores de rabiças que, segundo Veiga et al. (2014a), são largamente empregados em propriedades de até 20 hectares.

A maioria dos tratores de rabiças utilizam motores monocilíndricos arrefecidos a água. Normalmente são empregados motores do ciclo diesel de 
quatro tempos com potência entre 4,5 e $13,5 \mathrm{~kW}$. A cilindrada varia entre 250 e $500 \mathrm{~cm}^{3}$ com rotação nominal entre 2000 e 3500 rpm (Márquez, 2012). Esta máquina é composta por um eixo de duas rodas onde o operador caminha na parte posterior, direcionando-a através de comandos situados nas suas rabiças. São utilizadas principalmente na preparação de canteiros e no transporte de carga, este último através da adaptação de carreta que possibilita condução na posição sentada.

O crescimento da frota de tratores agrícolas incrementou o número e a gravidade dos acidentes no campo. Segundo Debiasi et al. (2004), a maior parte dos acidentes é causada por atitudes inseguras, sendo que a principal é a perda de controle em aclive ou declive, seguida da operação em condições extremas. Veiga et al. (2014b), analisando os tratores de rabiças, constataram que estes apresentam uma característica singular chamada 'inversão dos comandos', o que potencializa o risco de acidente. Na subida, ao acionar-se a embreagem de direção da roda esquerda, o veículo direciona-se para a esquerda. Já na descida, ao acionar-se a embreagem esquerda o veículo movimenta-se bruscamente para a direita. De acordo com OIT (2004), a agropecuária está entre as três atividades com maior índice de acidentes no mundo, sendo que para cada três acidentes ocorridos no meio rural, um causa a incapacidade permanente do trabalhador. Estudo realizado por Monteiro (2010) concluiu que 55\% dos acidentes graves ocorridos na zona rural do oeste paulista foram com máquinas agrícolas e dentre estas o trator foi o que causou $65 \%$ dos acidentes graves. A falta de atenção durante a operação foi a causa de $50 \%$ dos acidentes. Poletto (2009) verificou que as situações de risco são ignoradas pelos agricultores, por considerarem situações comuns na rotina de trabalho. Relata acidentes, de moderados a graves, como cortes em mãos, dedos e pernas. Situações estas vistas como normais por eles, que atribuem estas ocorrências à distração, falta de atenção na execução da tarefa e à falta de equipamento de proteção individual.

A operação de máquinas agrícolas exige uma carga mental elevada uma vez que são executados diversos processos com ajustes e regulagens específicas (Vilagra, 2009). As condições climáticas e ambientais adicionam fator de risco de acidente a esta atividade. O ruído, a vibração e a disposição espacial do posto de trabalho também têm influência na operação segura da máquina. Tanto Pawar (1978) como Veiga et al. (2015) destacam que a excessiva vibração e nível de pressão sonora são deficiências importantes nos projetos de tratores de rabiças. Mitarai et al. (2008a), analizando 76 operadores de tratores de rabiças, apontam a necessidade de melhoria do design do assento. Mitarai et al. (2008b), em estudo realizado nas Filipinas com 124 operadores de tratores de rabiças em operações em pé, constataram que um dos principais problemas apontados pelos operadores é o excessivo ruído do motor. Bidgoli et al. (2005) destacam que o trator de rabiças também realiza o transporte de passageiros porém, os efeitos do ruído sobre os condutores e passageiros não foram investigados de forma adequada. Quanto à disposição do posto de trabalho sentado, as distâncias dos comandos, incluindo alavanca de troca de marchas, embreagem e freio, excedem as distâncias ideais e máximas orientadas por Iida (2005), Grandjean (1998) e Dul \& Weerdmeester (2012), o que torna mais difícil sua condução. Veiga et al. (2015) compararam a usabilidade de comandos de direção de um trator de quatro rodas com a de um trator de rabiças. A simulação mostrou que o erro do trator de quatro rodas foi de $5,7 \%$ enquanto que para o trator de rabiças foi de $49,1 \%$.

O presente trabalho teve como objetivo identificar as principais causas de acidentes e suas correlações com fatores humanos e ambientais, ocorridos com tratores de rabiças em Santa Catarina.

\section{MATERIAL E MÉTODOS}

A pesquisa foi realizada nos anos de 2013 e 2014 em municípios pertencentes às mesorregiões Vale do Itajaí, Oeste Catarinense, Norte Catarinense e Serrana. Sua natureza exploratória teve como objetivo proporcionar maior familiaridade com o tema e constituir hipóteses. Já a característica descritiva permitiu o estabelecimento de relações entre variáveis humanas e ambientais. Utilizou-se o levantamento (survey) para melhor conhecimento do local (município), perfil dos acidentados (sexo e idade), dos tratores de rabiças envolvidos (tempo de uso e faixa de potência) e condições em que ocorreram estes acidentes. Além disso, o questionário semiestruturado explorou a causa do acidente, divididas em causas gerais (atitude ou condição insegura) e causas específicas. Como opções para as causas específicas foram utilizadas as mesmas propostas por Debiasi et al. (2004), com a adaptação da palavra 'trator' para 'trator de rabiças', a citar: falta de atenção, falha mecânica, operação do trator de rabiças em condições extremas, consumo excessivo de álcool, perda de controle aclive/declive, permissão de carona, falta de proteção, aproximação excessiva do trator de rabiças ligado, engate inadequado, entrada brusca do trator de rabiças em movimento, entrada involuntária do trator de rabiças em movimento e outras.

Para definição da amostra, foi utilizado o banco de dados de uma instituição federal de ensino agrícola. Dos 91 cadastros levantados (de famílias que declararam pertencer à agricultura familiar e possuir ao menos um trator de rabiças) foram sorteados 79 , seguindo metodologia para tamanho da amostra proposta por Barbetta (2010), com erro amostral tolerável de $4 \%$. Todos os sorteados residiam no estado de Santa Catarina. Os questionários foram enviados aos chefes de família e todos os entrevistados foram informados sobre o tema, os objetivos e os compromissos éticos da pesquisa, tendo sido assinado por estes um termo de consentimento livre e esclarecido.

Empregou-se nas frequências absolutas, para adequação do ajustamento, o método do qui-quadrado (qui-quadrado de Pearson) tanto na distinção do sexo (masculino ou feminino) como nas causas gerais dos acidentes (atitude insegura ou condição insegura).Os dados quantitativos foram tratados por correlação de Pearson que, segundo Barbetta (2010), é apropriado para des- 
crever a correlação linear dos dados de duas variáveis.

\section{RESULTADOS E DISCUSSÃO}

\section{Caracterização da amostra}

As cidades mais citadas foram Salete, São Joaquim, Alfredo Wagner, Rio do Campo, Ituporanga, Trombudo Central, Witmarsum, Chapadão do Lageado, $\mathrm{Pa}-$ panduva e Urupema, predominando os municípios pertencentes à mesorregião do Vale do Itajaí.

Nos municípios estudados, predomina a horticultura em regime familiar, que se desenvolve em pequenas propriedades, em sua maioria até 10 hectares. Os produtos mais relevantes são a cebola e o fumo. As máquinas pequenas, como os tratores de rabiças são as principais fontes de potência nestes empreendimentos rurais. O perfil do acidentado é do sexo masculino $(97,4 \%)$, com média de idade de 31,78 anos (desvio-padrão de 16,03 anos). As diferenças entre as frequências absolutas do sexo (masculino ou feminino) foram estatisticamente significativas, segundo o teste do qui-quadrado de Pearson a $1 \%$ de probabilidade de erro.

\section{Causas gerais dos acidentes segun- do os entrevistados}

Para 60,8\% dos entrevistados, o acidente relatado ocorreu por atitude insegura do trabalhador. Neste caso, mesmo conhecendo o risco da operação, o trabalhador se expôs, gerando o acidente. Para 30,4\% dos entrevistados a falha ocorreu por condição insegura, ou seja, deficiências no ambiente ou no trator de rabiças levaram à ocorrência do acidente. Para 8,8\% dos entrevistados a causa geral do acidente não foi identificada.

As diferenças entre as frequências absolutas das causas genéricas (atitude ou condição insegura) foram estatisticamente significativas, para ambas as classes de acidentes, segundo o teste do qui-quadrado de Pearson a $1 \%$ de probabilidade de erro.

Por esta análise observou-se resultado similar ao encontrado por Debiasi et al. (2004) quando estudou acidentes com diversos tipos de máquinas agrícolas, incluindo o trator de rabiças.

Devido a grande diferença entre a idade média dos acidentados $(31,78$ anos) e a idade média dos agricultores da região do Alto Vale, que segundo Poletto (2009), era de 48,9 anos (com desvio-padrão de 14,4 anos) investigouse a influência da idade do operador na ocorrência de determinado tipo de acidente. Foram avaliadas correlações entre a idade do acidentado e as causas gerais (atitude ou condição insegura) e posteriormente com as causas específicas mais citadas.

Correlação entre a idade do acidentado e a causa geral 'atitude insegura'

Para esta análise foram descartadas as faixas etárias de 0 até 10 anos. Isto por considerar que a vítima do acidente não teria clara distinção sobre os riscos que corria e, portanto, não caracterizaria ati- tude insegura. $\mathrm{O}$ cálculo do coeficiente de correlação de Pearson demonstrou uma tendência fortemente negativa entre a faixa etária e a atitude insegura, ou seja, quanto maior a idade, menor o número de acidentes. Considerando que a média de idade dos agricultores familiares da amostra (31,78 anos) envolvidos em acidentes é bem inferior à media de idade dos agricultores (48,9 anos) estudada por Poletto (2009); conclui-se que os jovens são os que se envolvem mais em acidentes com o trator de rabiças. Destaca-se a grande quantidade de acidentados acima de 10 até 20 anos, ocorrendo em $28 \%$ dos acidentes. Esse número sugere que menores de 18 anos estavam operando o trator de rabiças e que estes jovens correm mais risco pela exposição através de atitudes inseguras do que agricultores mais experientes (Tabela 1).

Correlação entre idade do acidentado e a causa geral 'condição insegura'

Analisando a ocorrência de acidentes cujo entrevistado julgou como causa geral a condição insegura, não identifica-se uma tendência quando comparada à faixa etária dos acidentados. As faixas acima de 20 até 30 anos e acima de 30 até 40 anos destacam-se por apresentar mais da metade dos relatos. A faixa etária e a condição insegura apresentou correlação negativa fraca, não significante para $\alpha=0,1 \%$. (Tabela 1 ).

\section{Causas específicas}

As causas específicas mais expressivas foram: falta de atenção $(28,0 \%)$,

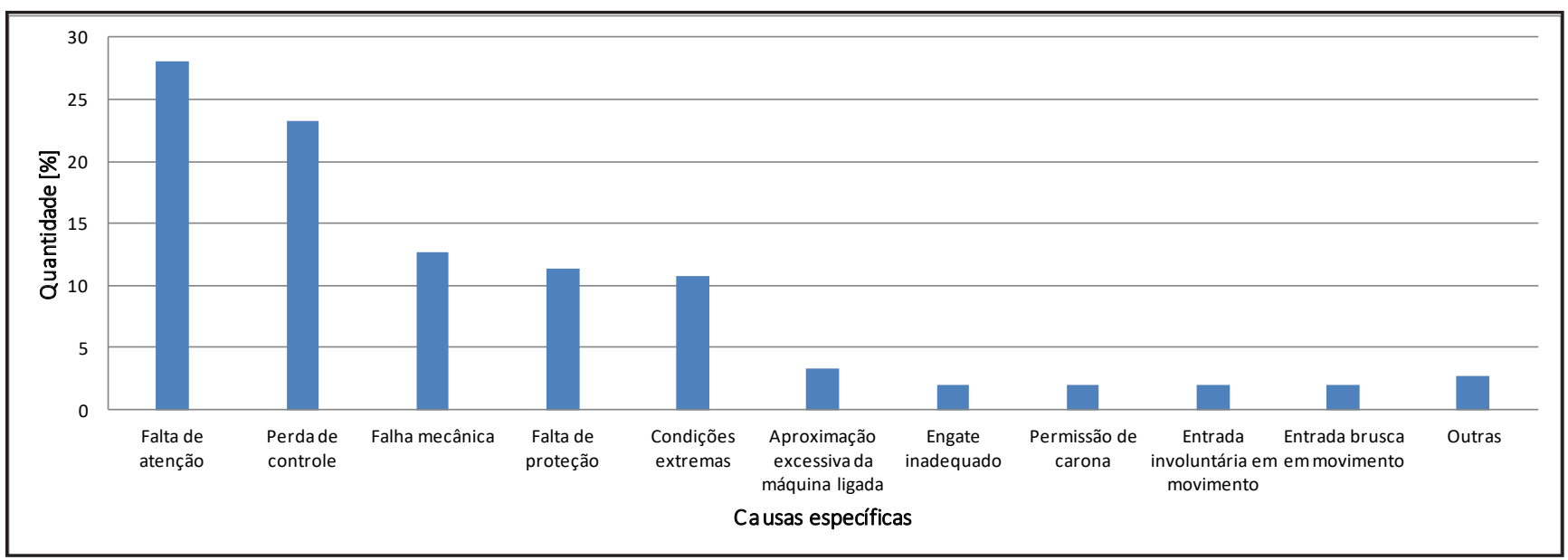

Figura 1. Causas específicas de acidentes mais citadas (most cited specific causes of accidents). Rio do Sul, IFC, 2014. 
Tabela 1. Correlações entre idade e as variáveis atitude insegura e condição insegura (correlation between age and unsafe attitude and between age and insecure condition). Rio do Sul, IFC, 2014.

\begin{tabular}{lccc}
\hline Variáveis & $\begin{array}{c}\text { Pearson } \\
(\mathbf{r})\end{array}$ & Correlação & $\begin{array}{c}\text { Significância } \\
(\boldsymbol{\alpha}=\mathbf{0 , 1 \% )}\end{array}$ \\
\hline Idade e atitude insegura & $-0,915$ & Negativa forte & $\mathrm{S}$ \\
Idade e condição insegura & $-0,224$ & Negativa fraca & $\mathrm{NS}$ \\
\hline
\end{tabular}

Em que $S=$ suficiente para afirmar a correlação (significance level sufficient to prove the correlation); NS= não suficiente para afirmar a correlação (significance level not enough to prove the correlation).

Tabela 2. Correlações entre idade e as variáveis falta de atenção, perda de controle, falha mecânica, falta de proteção e condições extremas (correlations between age and the variables inattention, loss of control, mechanical failure, lack of protection and extreme conditions). Rio do Sul, IFC, 2014.

\begin{tabular}{lccc}
\hline Variáveis & $\begin{array}{c}\text { Pearson } \\
(\mathbf{r})\end{array}$ & Correlação & $\begin{array}{c}\text { Significância } \\
(\boldsymbol{\alpha}=\mathbf{0 , 1 \% )}\end{array}$ \\
\hline Idade e falta de atenção & $-0,834$ & Negativa forte & $\mathrm{S}$ \\
Idade e perda de controle & $-0,621$ & Negativa moderada & $\mathrm{S}$ \\
Idade e falha mecânica & 0,756 & Positiva forte & $\mathrm{S}$ \\
Idade e falta de proteção & $-0,658$ & Negativa moderada & $\mathrm{S}$ \\
Idade e condições extremas & $-0,435$ & Negativa moderada & $\mathrm{S}$ \\
\hline
\end{tabular}

Em que $S=$ suficiente para afirmar a correlação (significance level sufficient to prove the correlation); $\mathrm{NS}=$ não suficiente para afirmar a correlação (significance level not enough to prove the correlation).

perda de controle em aclive/declive (23,3\%), falha mecânica $(12,7 \%)$, falta de proteção $(11,3 \%)$ e utilização em condições extremas (10,7\%) (Figura 1).

As duas primeiras causas estão ligadas a fatores humanos e cognitivos, assim como à carga de trabalho. Representam $51,3 \%$ dos acidentes e reforçam a idéia de que a maioria dos acidentes ocorre por atitude insegura.

A falta de atenção $(28,0 \%)$ pode estar associada ao excesso de carga mental, conforme relata Vilagra (2009) ou assumida pelo entrevistado por sentir-se culpado pelo acidente, como relata Poletto (2009).

A perda de controle em aclive/ declive $(23,3 \%)$, como segunda causa, corrobora com Veiga et al. (2014b) que apresentam a peculiaridade da inversão dos comandos, aumentando a possibilidade de erro de acionamento.

A falha mecânica $(12,7 \%)$ pode estar associada ao elevado tempo de uso dos tratores de rabiças presentes nas regiões.

A falta de proteção (11,3\%) pode ser evidenciada em situações de trabalho das apresentaram correlação moderada ou forte e foram significantes para $\alpha=$ $0,1 \%$ (Tabela 2).

\section{Correlação entre faixa etária e} falta de atenção

Encontrou-se uma correlação negativa forte entre estas variáveis. Pode ser explicada pela pouca experiência, que levaria o operador a subestimar os riscos aos quais está exposto na operação do trator de rabiças. A falta de atenção em momentos como a partida, trocas de marcha, frenagem e uso do trator de rabiças como fonte de potência podem ser elencadas como situações críticas para a ocorrência do acidente.

Correlação entre faixa etária e perda de controle em aclive/declive

A correlação negativa moderada encontrada, similarmente como ocorre com a falta de atenção, pode estar associada à pouca experiência, o que leva o operador a submeter o trator de rabiças às condições de declividade potencialmente perigosas. $\mathrm{O}$ trator de rabiças não dispõe de dispositivo que alerta quanto ao ângulo limite de operação (inclinômetro). Além disso, é comum na região do Alto Vale, a utilização de carreta tracionada, que transforma o trator de rabiças em trator com tração nas quatro rodas. A inversão dos comandos quando se passa de um aclive para declive e vice-versa constitui outro fator de risco de acidentes, mesmo entre operadores experientes.

\section{Correlação entre faixa etária e falha mecânica}

A correlação positiva forte pode ser justificada pelo excesso de confiança no trator de rabiças associado ao elevado tempo de uso da frota. Pelo levantamento, o ano de fabricação médio foi 1981, com desvio-padrão de 6,15 anos. Ou seja, o tempo de uso médio destas máquinas é acima de 30 anos.

\section{Correlação entre faixa etária e falta de proteção}

A correlação negativa moderada indica que quanto maior a idade do operador, menor foi a ocorrência de acidentes em função da não-utilização de proteção. Isto sugere que os mais experientes usavam com maior frequência os equipamentos de proteção individual do que os novatos. As partes do corpo 
lesionadas mais citadas foram mãos, pés, cabeça e tórax, corroborando com Poletto (2009). As lesões nas mãos estão associadas as operações de partida, acoplamento e desacoplamento de implementos, limpeza e manutenção do trator de rabiças. O uso de luvas de couro poderia reduzir o grau das lesões em muitos destes acidentes.

Correlação entre faixa etária e condições extremas

A correlação negativa fraca pode ser explicada pela inexperiência do operador e intenção de aumento de produção, levando o trator de rabiças a limites críticos de velocidade, rotação, carga ou declividade ou até mesmo associando dois fatores simultaneamente. Modificações no trator de rabiças ou na carreta foram evidenciadas no questionário, que poderiam justificar o aumento do risco de perda de controle ou capotamento.

Concluindo, o trator de rabiças continua sendo uma das máquinas mais utilizadas pelos pequenos horticultores das regiões Alto Vale do Itajaí, Oeste Catarinense, Norte Catarinense e Serrana. Sua operação pode gerar lesões que vão de leve a grave, inclusive levando a óbitos. A principal causa dos acidentes com trator de rabiças é a atitude insegura. Como causas específicas, em ordem decrescente, estão a falta de atenção, perda de controle em aclive/declive, falha mecânica, falta de proteção e seu uso em condições extremas. $\mathrm{O}$ estudo alerta quanto a importância da conscientização dos riscos na utilização dos tratores de rabiças, principalmente para os jovens, que estão sendo os mais afetados pelo uso inadvertido desta máquina.

\section{AGRADECIMENTOS}

Ao Instituto Federal Catarinense (IFC) e à Universidade Federal de Santa Catarina (UFSC) pela disponibilização de laboratórios, apoio financeiro e concessão de bolsa de estudo para a realização do projeto.

\section{REFERÊNCIAS}

ANFAVEA - Associação Nacional dos Fabricantes de Veículos Automotores. 2014. São Paulo: Anuário da Indústria Automobilística Brasileira. 154p.

BARBETTA, PA. 2010. Estatística aplicada às ciências sociais. 7a ed. Florianópolis: Editora UFSC. 340p.

BIDGOLI, SR; GHOBADIAN, B; NASSIRI P; KAMALIAN, N. 2005. Investigation, analysis and presentation of prediction models of a power tiller noise, pulling a trailer at rural asphalt road. Journal of Science and Technology of Agriculture and Natural Resources. Disponível em http://jstnar.iut. ac.ir/browse.php?a_code=A-10-2-316\&slc lang=en\&sid=1\&sw=River. Acessado em 19 de dezembro de 2015.

DEBIASI, H; SCHLOSSER, JF; WILLES, JA. 2004. Acidentes de trabalho envolvendo conjuntos tratorizados em propriedades rurais do Rio Grande do Sul, Brasil. Ciência Rural. Disponível em: <http://www.scielo.br/pdf/ cr/v34n3/a19v34n3.pdf>. Acessado em 15 dez. 2014.

DUL, J; WEERDMEESTER, B. 2012. Ergonomia prática . São Paulo: Edgard Blucher. 163p.

FAO/INCRA. 1994. Diretrizes de política agrária e desenvolvimento sustentável para a pequena produção familiar. Brasília.

GRANDJEAN, E. 1998. Manual de Ergonomia. Adaptando o trabalho ao homem. Porto Alegre: Bookman. 338p.

IIDA, I. 2005. Ergonomia, projeto e produção. São Paulo: Edgard Blucher. 614p.

MÁRQUEZ, L. 2012. Tractores agrícolas: tecnología y utilización. Madrid: Editora B\&H. 844p.

MITARAI, M; SICAT, JCV; KINOSHITA, O; TOYOMITSU, Y. 2008a Survey of riding tractor operating comfort in Nueva Ecija, Philippines. Japanese Journal of Farm Work Research. Disponível em https://www.jstage. jst.go.jp/article/jsfwr/43/2/43_2_67/_article. Acessado em 12 de janeiro de 2015 .

MITARAI, M; SICAT, JCV; KINOSHITA, O; TOYOMITSU, Y. 2008b. Survey of walking tractor operating comfort in Nueva Ecija, Philippines. Japanese Journal of Farm Work Research. Disponível em https://www.jstage. jst.go.jp/article/jsfwr/43/2/43_2_59/_article/ references. Acessado em 16 de janeiro de 2015.

MONTEIRO, LA. 2010. Prevenção de acidentes com tratores agrícolas e florestais. Botucatu: Editora Diagrama. 105p.

ORGANIZAÇÃO INTERNACIONAL DO TRABALHO (OIT) 2004. Panorama laboral. Oficina Regional para América Latina e Caribe.

PAWAR, JG. 1978. Investigation of human energy requirements for power tiller operation. India: Punjab Agricultural University. 172p. (Tese doutorado).

POLETTO, AR. 2009. Processo de trabalho e saúde mental de trabalhadores agrícolas familiares da microrregião de Ituporanga, Santa Catarina. Florianópolis: UFSC. 212p. (Tese doutorado).

VEIGA, RK; GONTIJO, LA; MASIERO, FC; VENTURI, J; ODORIZZI, W. 2014a. Emprego da análise ergonômica do trabalho em atividade com máquina agrícola motorizada. Exacta 12: 123-136.

VEIGA, RK; MASIERO, FC; GONTIJO, LA; ODORIZZI, W. 2014b. Multitarefas. Cultivar Máquinas 12: 6-9.

VEIGA, RK; MERINO, EAD; GONTIJO, LA; MASIERO, FC; MERINO, GSAD. 2015. Estudo comparativo da usabilidade de comandos direcionais para duas concepções de máquinas agrícolas. Revista Produção Online 15: 830-858.

VILAGRA, JM. 2009. Adequação ergonômica de trator agrícola de média potência: construção e validação de um instrumento de avaliação a partir do construto de conforto, segurança e eficiência. Florianópolis: UFSC. 133p. (Tese doutorado). 